Article

\title{
Effects of Plyometric Training on Surface Electromyographic Activity and Performance during Blocking Jumps in College Division I Men's Volleyball Athletes
}

\author{
Min-Hsien Wang ${ }^{1}$, Ke-Chou Chen ${ }^{2}$, Min-Hao Hung ${ }^{3}$, Chi-Yao Chang ${ }^{3}$, Chin-Shan Ho ${ }^{3}{ }^{1}$, \\ Chun-Hao Chang ${ }^{3}$ and Kuo-Chuan Lin ${ }^{2, *}$ \\ 1 Department of Physical Education, Chinese Culture University, Taipei 11114, Taiwan; \\ samwang6601@gmail.com \\ 2 Office of Physical Education, Chung Yuan Christian University, Taoyuan 320314, Taiwan; ivan@cycu.edu.tw \\ 3 Graduate Institute of Sports Science, National Taiwan Sport University, Taoyuan 333325, Taiwan; \\ a2822180@gmail.com (M.-H.H.); anthonychang1124@gmail.com (C.-Y.C.); kilmur33@gmail.com (C.-S.H.); \\ hao781106@gmail.com (C.-H.C.) \\ * Correspondence: misvb@cycu.edu.tw
}

Received: 29 April 2020; Accepted: 29 June 2020; Published: 30 June 2020

\begin{abstract}
In volleyball matches, there are three minute intervals between sets. Therefore, the improvement of the muscle output ratio is one of the most import foundational physical elements for the players. The purpose of this study was to investigate the effects of plyometric training on the changes in electrical signals in the lower limb muscles of male college volleyball players during continuous blocking and to examine the benefits of plyometric training on blocking agility and maximum vertical jump height. In this study, twenty elite male college volleyball players were recruited and divided into a plyometric training group (PTG) and a control group (CG). The wireless electromyography was used for data acquisition, and the electrodes were applied to the left and right rectus femoris, biceps femoris, tibialis anterior, and gastrocnemius. The median frequency was used as the measurement of the electromyographic signals during the jumping blocks. This study used covariate analysis methods, with previously measured results used as covariates to perform a two-way analysis of covariance for the independent samples. Based on the results of this study, after 6 weeks of training, the median frequency of the rectus femoris ( $2.13 \%$ to $4.75 \%$ improved) and that of the tibialis anterior muscles (4.14\% to $7.71 \%$ improved) were significantly lower in the PTG than in the CG. Additionally, the blocking agility increased by $6.26 \%$ and the maximum vertical jump height increased by $3.33 \%$ in the PTG compared to the CG. The findings provide important insights on the neuromuscular status for volleyball players during continuous blocking jumps. Six weeks of appropriate plyometric training can facilitate the performance of volleyball players.
\end{abstract}

Keywords: muscular activity; blocking agility; maximum vertical jump height; median frequency

\section{Introduction}

Jumping ability is essential for performance in volleyball. Superior rebounding not only enables players to gain a competitive advantage on offense (increasing blocking height and attack angle) but also allows for a larger defensive range [1-3]. To increase the vertical jumping ability, weight-bearing jumping or plyometric jump-training methods can effectively improve leg muscles and explosive power, thereby improving overall strength and coordination in the legs. Vertical jumping is an important basic skill in many sports [4], but repetitive jumping is the primary cause of muscle fatigue. A study 
by Merletti and Parker [5] showed that the sites of neuromuscular fatigue can be divided into the following three major categories: central fatigue, fatigue of the neuromuscular junction, and muscle fatigue. Power-generating nerve signal transmission can be divided into central and peripheral nerve levels; thus, fatigue can be classified as central fatigue or peripheral fatigue based on the different sites of fatigue. Decreased muscle strength during fatigue is accompanied by central and peripheral effects. The former is associated with a decrease in the number of motor units involved in the action or a reduction in the frequency at which motor units are evoked.

It is well known that fatigue caused by vertical jumping can alter muscular characteristics, reduce muscle effectiveness, and change the maximum joint torque [6,7]. However, superior vertical jumping ability requires an excellent coordination of movements, that is, the ability to control and adjust musculoskeletal characteristics [8]. Therefore, fatigue may be a factor that causes changes in the central nervous system (CNS) and alters the coordination of the limbs during vertical jumps. The effects of post-jump fatigue on motor coordination have been discussed in previous studies [9-11], and the results showed that continuous jumping can reduce knee and ankle strength. Additionally, in the case of decreased muscle strength, it is not possible to improve strength even if the range of motion of the lower limb joints is increased.

Previous studies [12] have indicated that the rectus femoris, vastus lateralis, vastus medialis, and biceps femoris are the primary muscles used during squat jumps. After 50 repeated squat jumps, the maximum voluntary contraction of the knee extensors decreases by $25 \pm 11 \%$, but there is no significant change in the knee flexors. In ve Dikey et al. [13], the correlation between maximum isokinetic strength, muscle activity, and jump height in 12 elite male volleyball players was investigated. The results of the study showed that hamstring/quadriceps ratios were greatest at an angular velocity of $240^{\circ} / \mathrm{s}$. Additionally, the degree of biceps femoris activity was greater than that of the vastus lateralis and vastus medialis regardless of the angular velocity, and the degree of activity of the biceps femoris and the rectus femoris was consistent. The degree of muscular activity (MVC\%) of the knee extensors and knee flexors increases with increasing fatigue. Therefore, some studies have recommended the evaluation of vertical jumping based on actual sports conditions [14,15]. In this method, individuals performed intermittent fixed-height jumps, such as intermittent jumps to $95 \%$ of the maximum vertical jump height, until the target jump height cannot be reached for three consecutive attempts. The degree of fatigue is estimated from the resting interval after each jump and the number of vertical jumps [14].

Plyometric training is a typical high-intensity exercise, and the generated peripheral fatigue can be significantly improved after plyometric training [16]. A main finding is that the maximum voluntary contraction and degree of activity generated by muscles are significantly improved after plyometric training [17]. Plyometric training can effectively enhance muscle activity and improve the muscle output ratio. This effect not only significantly increases the maximum jump height but also enhances the coordination of the lower limb muscles [18]. Plyometric training is a jumping training method that uses the physiological phenomenon of a stretch shortening cycle (SSC) to produce stronger contractions during the centripetal contraction phase [19]. Because volleyball players must perform movements such as repeatedly jumping, sprinting and changing direction, this training method is appropriate for targeting their physical training needs and is widely applicable $[20,21]$.

According to previous studies, plyometric training has many positive effects on volleyball players. However, most of the prior studies focused on improvements in general physical abilities due to plyometric training. In this study, sport-specific tests were evaluated with game-like situations simulating volleyball blocking jumps. The tests required repetitions of blocking jumps, during which the fatigue state of the lower limb muscles was recorded. The measurement methods used in this study were modified from the study by Sheppard et al. [1], which used repetitive jumping blocks in competition-like conditions. Surface electromyography with electrodes attached to the lower limb muscle groups were used to elucidate the mechanisms and conditions caused by muscle fatigue. Plyometric training was used to strengthen muscle groups prone to fatigue so as to reduce the rate at 
which fatigue develops and enhance athletic performance. The initial hypothesis of this study was that plyometric training can induce positive changes in muscle activities in male college volleyball players.

\section{Methods}

\subsection{Participants}

This study was designed to be similar to a blocking action situation in actual competition. Players were divided into a plyometric training group (PTG) and a control group (CG) during the training period. Before entering the training cycle, the players in both groups underwent a pre-test. The PTG underwent six weeks of training after the pre-test, and the CG maintained the original training program. The two groups underwent a post-test after six weeks. In this study, 20 elite male college volleyball players were used as the study participants, and they were equally divided into the PTG $(\mathrm{n}=10$; mean age $=21.5 \pm 1.2$ years; mean height $=186.5 \pm 5.1 \mathrm{~cm}$; mean weight $=78.1 \pm 4.7 \mathrm{~kg})$ and the $C G(\mathrm{n}=10$; mean age $=22.1 \pm 1.5$ years; mean height $=176.5 \pm 4.4 \mathrm{~cm}$; mean weight $=77.4 \pm 5.2 \mathrm{~kg}$ ). All the participants in the study had participated in professional volleyball training for over five years and were registered in the men's Division I of the Republic of China University Volleyball League. The subjects were free of major musculoskeletal system disorders within the year preceding the study. This study conducted experiments using competition-like conditions during testing. Although slightly different from the actual competition conditions, verbal cues were used in the experiment, and the participants were required to make their best effort. All subjects gave their informed consent for inclusion before they participated in the study. The study was conducted in accordance with the Declaration of Helsinki, and the protocol was approved by the Ethics Committee of Fu Jen Catholic University Institutional Review Board (C103117).

\subsection{Data Collection}

In this study, 10 Vicon T40 motion capture systems (Vicon MX-Giganet, Oxford Metrics Ltd., UK) and Nexus software (Version 4.0.2.; Vicon Motion System Ltd, UK) were used with a frame rate of $250 \mathrm{~Hz}$. One marker was attached on the seventh cervical vertebra (C7) to assess the jump height. In this study, wireless electromyography (BTS Free EMG; BTS Bioengineering Corp., USA) was used by attaching electrodes to muscle groups in the left and right legs, including the rectus femoris (RFM), biceps femoris (BFM), tibialis anterior (TAM), and gastrocnemius (GM) [22]. The sampling frequency was set to $1000 \mathrm{~Hz}$. According to previous research [22], five muscles, each with their own specific anatomical function, have the major influences on jumping movements, as follows: RFM (hip joint flexion, knee joint extension), BFM (hip joint extension, knee joint flexion), vastus lateralis muscle (knee joint extension), GM (knee joint flexion, ankle plantar flexion), and TAM (ankle dorsiflexion, inversion). In this study, the athletes were required to perform blocking jumps in two directions for a total of 14 times. We aimed to observe the muscles that dominated in at least two functions during the blocking jumps. Therefore, the EMG measurements of the RFM, BFM, TAM, and GM were recorded. The blocking agility system $(1000 \mathrm{~Hz}$ sampling frequency) was used to record the total response time [23].

\subsection{Experimental Design}

Actual testing was performed as modified in the study by Sheppard et al. [1]. The blocking target was placed three meters away from the starting position of the movement, and the blocking height was set to $90 \%$ of the subject's individual jump height. The subjects performed 14 moving blocks. A total of three groups of tests (T1, T2, T3) were performed, with a three-minute rest between the groups. To give the subjects a fixed load, the blocking agility (BA) test from a study by Ho et al. [23] was used, with an eight-second interval between the blocks. All the data in this study were collected simultaneously using the Vicon server. The site layout is shown in Figure 1. 


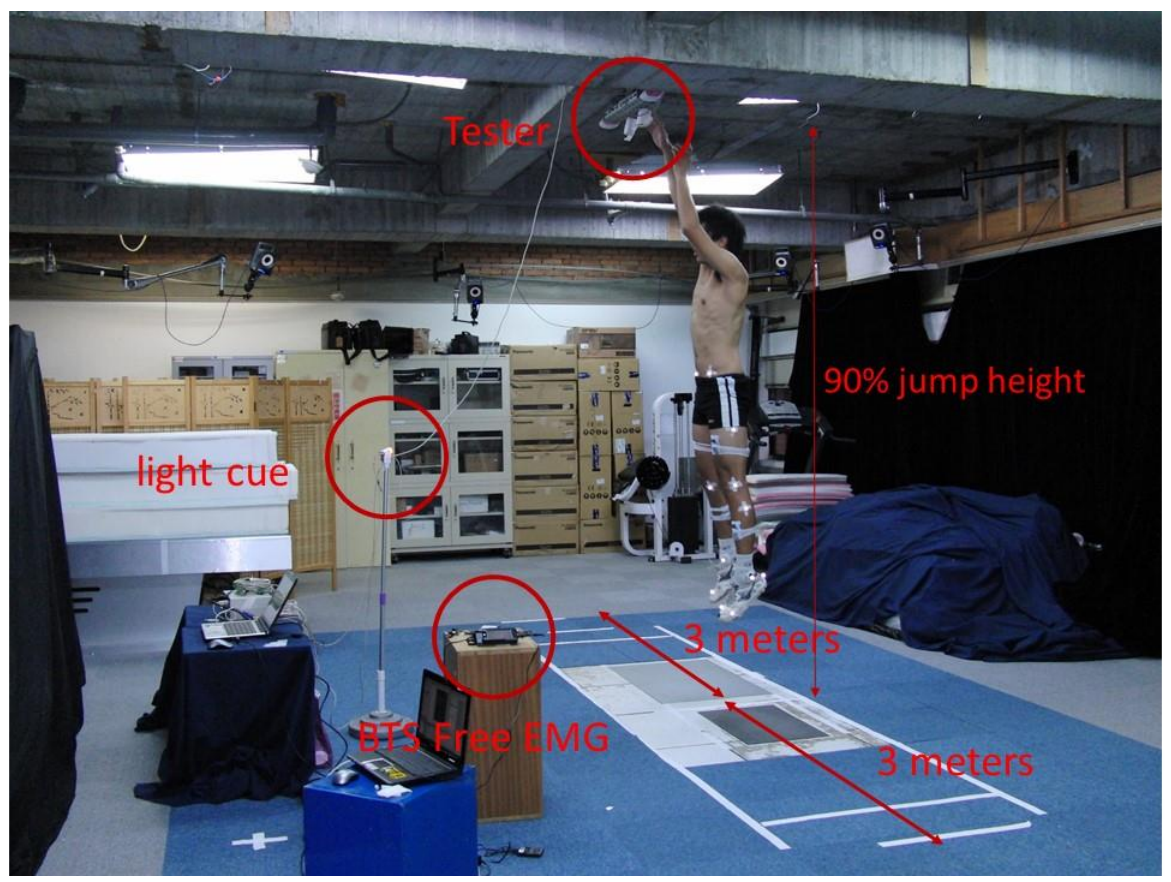

Figure 1. Diagram and explanation of the blocking agility test for volleyball. The athlete was required to perform a total of 14 blocks from two directions. The blocking agility test system and wireless electromyography (BTS Free EMG) were set in the front of the athlete.

\subsection{Procedures}

Before the start of the experiment, the participants were collectively informed about the purpose of the study and the experimental process, and any issues needing attention were addressed. Additionally, their rights during the study were disclosed, after which the subjects gave written informed consent. One week before testing, all the participants were provided with information about the experiment and administered two practice tests. During the course of the experiment, the subjects were instructed to not change their daily training program or training volume. The PTG underwent six weeks of plyometric training during the study. The primary purpose of their regimen was to train the muscle groups in the legs. The training program for this study was formulated as described by Makaruk and Sacewicz [18] (Table 1). During the six-week plyometric training program, the subjects participated in training sessions twice a week. The intensity and training volume of the regimen was based on the number of groups and repetitions proposed by Piper and Erdmann [24]. Two weeks of medium-intensity training and four weeks of high-intensity training were implemented in the program to improve the transmission of the CNS signals in the subjects and avoid excessive load or fatigue. 
Table 1. Plyometric training program (PTG).

\begin{tabular}{|c|c|c|c|}
\hline $\begin{array}{l}\text { Plyometric Training Program } \\
\text { Number of Sets } \times \text { Number of Rebounds }\end{array}$ & $\begin{array}{c}\text { Week 1-2 } \\
(\text { Sets } \times \text { Rebs) }\end{array}$ & $\begin{array}{c}\text { Week 3-4 } \\
\text { (Sets } \times \text { Rebs) }\end{array}$ & $\begin{array}{c}\text { Week 5-6 } \\
(\text { Sets } \times \text { Rebs) }\end{array}$ \\
\hline Standing vertical hops & $2 \times 10$ & & \\
\hline Single foot hops & $4 \times 8$ & & \\
\hline Multiple two-foot hurdle jumps (hurdle height $0.55 \mathrm{~m}$ ) & $6 \times 6$ & & \\
\hline Counter movement jumps & $3 \times 5$ & & \\
\hline Depth jumps (drop box height $0.20 \mathrm{~m}$ ) & $3 \times 6$ & & \\
\hline Lateral two-foot jumps & & $2 \times 10$ & \\
\hline Two-foot jumps & & $4 \times 8$ & \\
\hline Counter movement jumps & & $3 \times 5$ & \\
\hline Multiple two-foot hurdle jumps (hurdle height $0.65 \mathrm{~m}$ ) & & $6 \times 6$ & \\
\hline Depth jumps (drop box height $0.30 \mathrm{~m}$ ) & & $3 \times 6$ & \\
\hline Two-foot jumps forward and backward: & & & $2 \times 10$ \\
\hline Single foot jumps & & & $2 \times 8$ on each foot \\
\hline Counter movement jumps & & & $3 \times 5$ \\
\hline Multiple two-foot hurdle jumps (hurdle height $0.76 \mathrm{~m}$ ) & & & $6 \times 6$ \\
\hline Depth jumps (drop box height $0.40 \mathrm{~m}$ ) & & & $3 \times 6$ \\
\hline
\end{tabular}

\subsection{Dependent Measures}

The parameters investigated in this study were the myoelectric signals, blocking agility (BA), and the maximum vertical jump height. The collection and processing of each parameter are described as follows:

1. Collection and processing of the maximum vertical jump height

The participants were asked to stand on a force plate to perform a countermovement jump, which was measured using a three-dimensional motion capture system. The maximum vertical jump height test was performed three times. A program was written in Matlab software (Version R2008a; The MathWorks Inc., USA) to calculate the height of the highest point after the player takes off.

2. Blocking agility (BA) test

The subjects were required to warm up adequately for 10-15 min, with special attention focused on extending the joint ligaments in the legs. The subjects could complete the warm-up when they felt comfortable and ready. In the test, the blocking agility system was used to produce visual stimuli and record the total response time during blocking. The examiner gave a voiced signal to the athlete and triggered the blocking agility test system at the same time. The light cue was activated as stimulation $8 \mathrm{~s}$ after the system was triggered. During the BA test, the subjects were instructed to land on both feet. The actions were performed seven times in each direction (left and right) for a total of 14 blocks, and three repeated sets of records were analyzed. The subjects were directed to stand in the preparation area, which was three meters away from the blocking point, and to wait for a light to turn on. When the light turned on, the subject completed the blocking action as quickly as possible by touching the target.

3. Collection and processing of the muscle median frequency

Free EMG was used to wirelessly transmit data to a computer, and the collected EMG data were stored and Matlab 7.0.1 was used to read and write a program. The EMG signals were band-pass filtered $(10-500 \mathrm{~Hz})$ with a fourth order Butterworth filter. The median frequency (MDF) was derived from the EMG data; MDF is the frequency value that divides the power spectrum into two equal regions and is considered a reliable method for assessing muscle fatigue during exercise [25]. Studies [26,27] have shown that when muscles become fatigued, high-frequency motor units are evoked less frequently, and low-frequency motor units are evoked more frequently and recruited in larger numbers. This increases the slow contraction effects and slows muscle fiber conduction velocity, such that the MDF of the power spectrum is biased towards low frequencies (Figure 2); this characteristic can be used to assess muscle fatigue. 



(a)

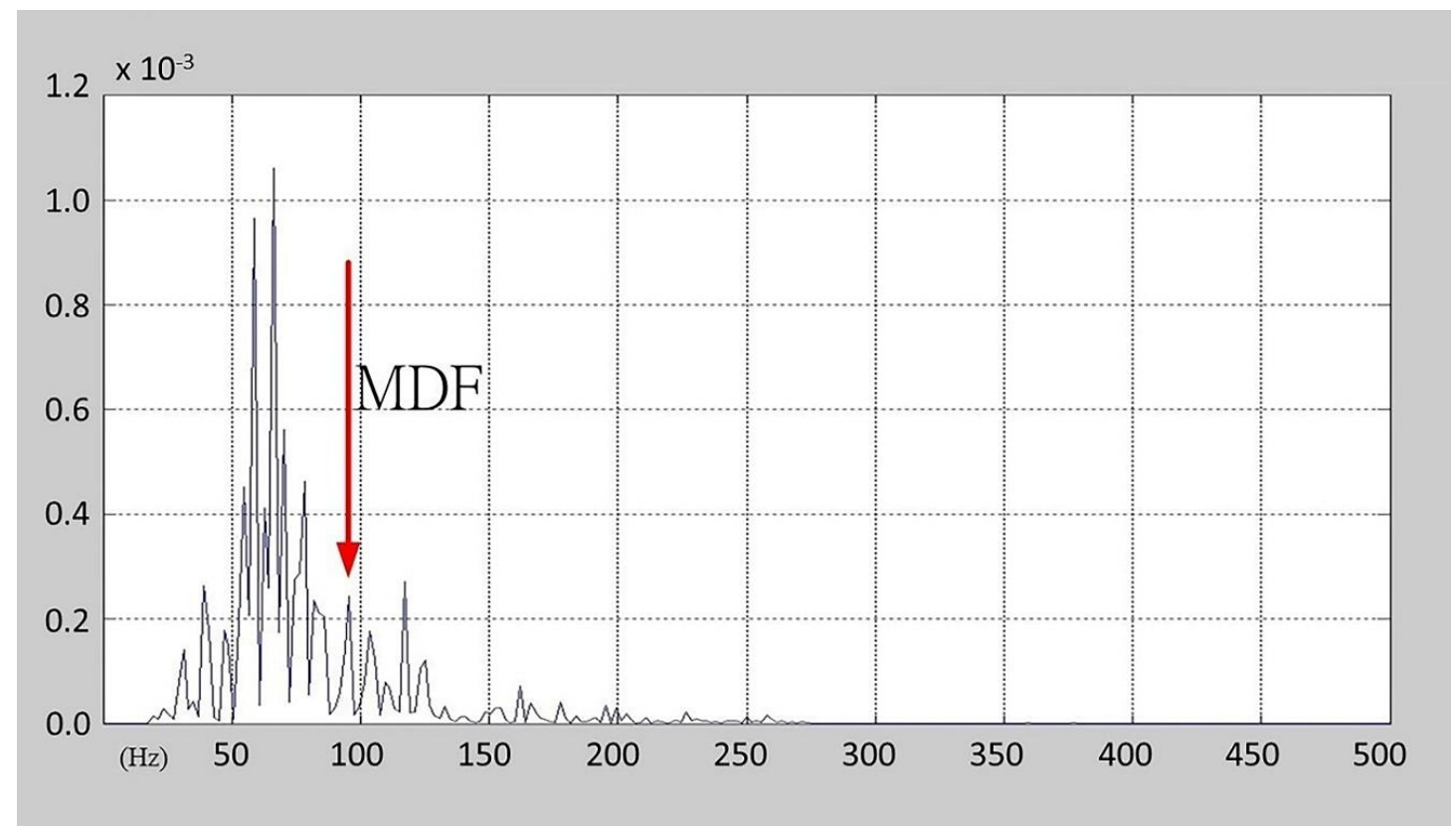

(b)

Figure 2. The signals of surface electromyographic activity and the results of median frequency (MDF) on the lower extremity muscles. (a) The EMG signals for the rectus femoris (RFM), biceps femoris (BFM), tibialis anterior (TAM), and the gastrocnemius (GM) during the blocking jumps. The time series of the EMGs were captured and analyzed between the subject contact "*" with the force-plate and when it leaves "o". (b)The median frequency of the signal spectrum.

\subsection{Statistical Analysis}

The Statistical Package for the Social Sciences (SPSS) 20.0 software (version 20.0; SPSS Inc., Chicago, IL, USA) was used for the statistics and data analysis. First, the reliability of the measured data was tested using the intra-class correlation coefficient (ICC). This study primarily investigated the effects of plyometric training and continuous blocking on the MDFs of the RFM, TAM, GM, and BFM of the study participants. Descriptive statistics methods were used to describe the median frequency of the participants tested before and after the blocking rounds. As some interfering factors in actual 
experimental situations can affect experimental results, a two-way analysis of covariance (ANCOVA) with pre-test performance as the covariate was used to correct for sources of error and increase the accuracy. If the effect of the two factors reached the level of significance, a simple effects test (Bonferroni post-hoc test) was performed. If the interaction did not reach the level of significance, a main effects test was performed. The significance level was set at alpha $\leq 0.05$.

\section{Results}

The pre- and post-test ICCs for the MDF (RFM, TAM, GM, and BFM) and jump performances are shown in Table 2. All the variables showed moderate to excellent results. ANCOVA, which was used in this study, requires that the variables in the data are homogeneous. The results of Levene's test showed that all the variables were homogeneous $(p>0.05)$ and that there were no interactions between the covariate and the independent variables that met the basic assumptions for ANCOVA.

Table 2. The pre- and post-test intra-class correlation coefficient (ICC) for the MDF (RFM, TAM, GM, and BFM) and jump performances (blocking agility (BA) and maximum vertical jump height).

\begin{tabular}{ccccccc}
\hline & \multicolumn{3}{c}{ MDF } & \multicolumn{2}{c}{ Jump Performances } \\
\cline { 2 - 6 } & RFM & TAM & GM & BFM & BA & $\begin{array}{c}\text { Maximum Vertical } \\
\text { Jump Height }\end{array}$ \\
\hline Pre-test & 0.991 & 0.976 & 0.614 & 0.660 & 0.973 & 0.964 \\
Post-test & 0.978 & 0.936 & 0.560 & 0.690 & 0.988 & 0.983 \\
\hline
\end{tabular}

\subsection{Muscle Activities of Blocking Jumps}

The results of pre- and post-testing in the PTG and CG (Table 3) show that as the number of rounds increased, the MDF of both the RFM and TAM decreased, but the MDFs of the GM and BFM did not. The frequency was highest in the BFM followed by the TAM; the frequencies of the RFM and GM were similar. The differences in the RFM reached the level of significance with respect to the group and group $\times$ round but not with respect to the round. The comparison of the marginal means showed that the PTG $>$ the CG. The differences in the TAM reached the level of significance with respect to the group and group $\times$ round but not with respect to the round. The comparison of the marginal means showed that the PTG > the CG. The differences in the GM did not reach the level of significance with respect to the group, round, or group $\times$ round. The differences in the BFM did not reach the level of significance with respect to the group, round, or group $\times$ round. As the interaction between the RFM and the TAM reached the level of significance with respect to the group $\times$ round, a simple main effects test was performed. The results for the RFM in the CG showed that the MDF decreased by $11.55 \%$ and $20.61 \%$ in the second and third rounds of the pre-test, respectively, and by $11.04 \%$ and $21.52 \%$ in the second and third rounds of the post-test, respectively, exhibiting similar declines. In the PTG, the MDF decreased by $13.49 \%$ and $22.42 \%$ in the second and third rounds of the pre-test, respectively, and by $11.36 \%$ and $17.67 \%$ in the second and third rounds of the post-test, respectively; the decrease in the MDF in the PTG was significantly slower than that in the CG. The results of the MDF for the TAM in the CG showed that the MDF decreased by $23.96 \%$ and $31.79 \%$ in the second and third rounds of the pre-test, respectively, and by $22.20 \%$ and $30.99 \%$ in the second and third rounds of the post-test, respectively; the degrees of decline in the MDF were nearly the same in both groups. In the PTG, the MDF decreased by $25.08 \%$ and $35.02 \%$ in the second and third rounds of the pre-test, respectively, and by $20.94 \%$ and $27.31 \%$ in the second and third rounds of the post-test, respectively. 
Table 3. Values are the mean \pm standard deviation. Data are reported for the MDF of the RFM, BFM, TAM and the GM during the blocking jumps. The unit of MDF is hertz $(\mathrm{Hz})$.

\begin{tabular}{|c|c|c|c|c|c|c|c|c|c|c|}
\hline & & \multicolumn{3}{|c|}{ PTG } & \multicolumn{3}{|c|}{ CG } & \multirow{2}{*}{$\begin{array}{c}\text { Interaction } \\
\text { Effect (F-value) }\end{array}$} & \multirow{2}{*}{$\begin{array}{l}\text { Simple Effects } \\
\text { (F-value) }\end{array}$} & \multirow{2}{*}{ Post-hoc Test } \\
\hline & & T1 & T2 & T3 & T1 & T2 & T3 & & & \\
\hline \multirow{2}{*}{ RFM } & Pre-test & $109.7 \pm 7.2$ & $94.9 \pm 3.7$ & $85.1 \pm 3.1$ & $108.2 \pm 6.4$ & $95.7 \pm 4.4$ & $85.9 \pm 3.9$ & \multirow{2}{*}{$23.624^{* *}$} & $\mathrm{~T} 1=0.301$ & \multirow{2}{*}{$\begin{array}{l}\text { PTG }>\text { CG } \\
\text { PTG }>\text { CG }\end{array}$} \\
\hline & Post-test & $109.2 \pm 6.6$ & $96.8 \pm 3.6$ & $89.9 \pm 3.5$ & $107.8 \pm 6.4$ & $95.9 \pm 3.7$ & $84.6 \pm 4.0$ & & $\begin{aligned} \mathrm{T} 2 & =8.136^{*} \\
\mathrm{~T} 3 & =40.690^{* *}\end{aligned}$ & \\
\hline \multirow{2}{*}{ BFM } & Pre-test & $200.4 \pm 9.5$ & $172.2 \pm 32.8$ & $172.4 \pm 27.3$ & $188.1 \pm 19.4$ & $174.8 \pm 35.8$ & $178.7 \pm 30.1$ & \multirow{2}{*}{1.639} & & \\
\hline & Post-test & $193.6 \pm 5.6$ & $174.3 \pm 27.7$ & $170.9 \pm 27.1$ & $187.4 \pm 18.6$ & $188.1 \pm 18.4$ & $183.1 \pm 27.8$ & & & \\
\hline \multirow{2}{*}{ TAM } & Pre-test & $182.2 \pm 7.7$ & $136.5 \pm 4.3$ & $118.4 \pm 7.2$ & $174.9 \pm 15.9$ & $133.0 \pm 2.9$ & $119.3 \pm 6.7$ & \multirow{2}{*}{$11.086^{* *}$} & $\mathrm{~T} 1=1.284$ & \multirow{2}{*}{$\begin{array}{l}\text { PTG }>\text { CG } \\
\text { PTG }>\text { CG }\end{array}$} \\
\hline & Post-test & $180.5 \pm 6.2$ & $142.7 \pm 6.4$ & $131.2 \pm 2.7$ & $170.7 \pm 17.7$ & $132.8 \pm 3.0$ & $117.8 \pm 7.6$ & & $\begin{array}{l}\mathrm{T} 2=95.390^{* *} \\
\mathrm{~T} 3=109.149^{* *}\end{array}$ & \\
\hline \multirow{2}{*}{ GM } & Pre-test & $101.1 \pm 28.9$ & $94.4 \pm 11.2$ & $100.9 \pm 14.2$ & $95.3 \pm 9.3$ & $95.5 \pm 8.1$ & $100.9 \pm 97.2$ & \multirow{2}{*}{1.093} & & \\
\hline & Post-test & $96.1 \pm 29.3$ & $87.5 \pm 14.0$ & $92.9 \pm 10.2$ & $92.0 \pm 21.4$ & $93.4 \pm 9.7$ & $99.1 \pm 9.1$ & & & \\
\hline
\end{tabular}

PTG: plyometric training group; CG: control group. The table indicates significant change (post-pre) when using the pre-test score as a covariate: ${ }^{*} p<0.05 ;{ }^{* *} p<0.01$. RFM: rectus femoris muscle; BFM: biceps femoris muscle; TAM: tibialis anterior muscle; GM: gastrocnemius muscle. 


\subsection{Jump Performances}

Table 4 shows the BA of the two groups measured before and after training. The average BA test score over the three rounds in the PTG and CG was $2.08 \pm 0.14 \mathrm{~s}$ and $2.09 \pm 0.14 \mathrm{~s}$ in the pre-test, respectively, and $1.95 \pm 0.11 \mathrm{~s}$ and $2.08 \pm 0.17 \mathrm{~s}$ in the post-test, respectively. This shows that the magnitude of decrease in the post-test was larger in the PTG than in the CG. The ANCOVA results showed that the BA test reached the level of significance with respect to the group but not with respect to the round or group $\times$ round $(p>0.05)$. Since there were only two levels of group factors, comparing the marginal means showed that the PTG > the CG; that is, the BA of the PTG was significantly higher than that of the CG. However, the results of the descriptive statistics showed that in the CG, the BA increased by $0.48 \%$ and $1.93 \%$ in the second and third rounds of the pre-test, respectively, and by $0.97 \%$ and $1.46 \%$ in the second and third rounds of the post-test, respectively, exhibiting similar increases. In the PTG, the BA increased by $1.96 \%$ and $3.43 \%$ in the second and third rounds of the pre-test, respectively, and by $1.04 \%$ and $1.55 \%$ in the second and third rounds of the post-test, respectively.

Table 4. Values are the mean \pm standard deviation. Data are reported for the blocking agility (BA) test. The unit of BA is second (s).

\begin{tabular}{|c|c|c|c|c|c|c|c|c|c|}
\hline & \multicolumn{3}{|c|}{ PTG } & \multicolumn{3}{|c|}{ CG } & $\begin{array}{c}\text { Interaction } \\
\text { Effect } \\
\text { (F-value) }\end{array}$ & $\begin{array}{c}\text { Main } \\
\text { Effects } \\
\text { (F-value) }\end{array}$ & $\begin{array}{c}\text { Post-hoc } \\
\text { Test }\end{array}$ \\
\hline Pre-test & $2.04 \pm 0.16$ & $2.08 \pm 0.13$ & $2.11 \pm 0.13$ & $2.07 \pm 0.15$ & $2.08 \pm 0.16$ & $2.11 \pm 0.13$ & 0.618 & $58.287^{* *}$ & CG $>$ PTG \\
\hline
\end{tabular}

Table 5 shows the maximum vertical jump height of the two groups measured before and after training. The average jump height in the PTG and CG were $67.04 \pm 3.83 \mathrm{~cm}$ and $66.86 \pm 4.06 \mathrm{~cm}$ in the pre-test, respectively, and $69.27 \pm 3.87 \mathrm{~cm}$ and $66.79 \pm 3.76 \mathrm{~cm}$ in the post-test, respectively. This shows that the PTG had a large magnitude of improvement after six weeks of plyometric training. There were significant differences between the groups. Comparing the marginal means showed that the PTG $>$ the $\mathrm{CG}$, that is, the plyometric training could effectively improve the maximum vertical jumping ability of the study participants.

Table 5. Values are the mean \pm standard deviation. Data are reported for the maximum vertical jump height. Units in centimeters $(\mathrm{cm})$.

\begin{tabular}{ccccc}
\hline & PTG & CG & F-value & Post-hoc Test \\
\hline Pre-test & $67.04 \pm 3.83$ & $66.86 \pm 4.06$ & $26.862 * *$ & PTG $>$ CG \\
Post-test & $69.27 \pm 3.87$ & $66.79 \pm 3.76$ & & \\
\hline
\end{tabular}

PTG: plyometric training group; CG: control group. The table indicates significant change (post-pre) with the pre-test score as a covariate: ${ }^{* *} p<0.01$.

\section{Discussion}

Based on the results of this study, the decrease in the MDF of the RFM and TAM was significantly lesser in the PTG after six weeks of training compared to that of the CG, and there was significant improvement in both the BA performance and maximum vertical jump height in the PTG.

From the results, the MDF increased in both the RFM and TAM as the number of rounds increased. In other words, under the effects of the exercise load in this study, the contraction ability of the muscles was reduced, and the characteristics of the power spectrum were altered. Among the MDF data for the four muscles, the TAM showed the greatest improvement. However, the MDF of these four muscles still decreased as the number of rounds in the blocking test increased. The amount of muscle recruitment in various parts of the legs when jumping was studied [22], and the results showed that the principal muscle groups recruited when performing squat jumps are the TAM $>$ RFM $>$ GM $>$ 
BFM; that is, the TAM and RFM are the principal muscle groups used when jumping. In the results of the continuous jump blocking test conducted on the study participants in the present study, the decrease in MDF in the legs improved in the PTG after a six-week interventional training; this result is consistent with that of previous studies [16]. The report [16] indicated that the plyometric training (PT) increased central fatigue significantly by about $15-20 \%$, but significantly decreased peripheral (muscle) fatigue during the 2-min MVC by about 10\% in the quadriceps femoris. In this study, the MDF of the RMF and TAM of the PTG and CG decreased by similar percentages in the pre-test. However, the MDF of the PTG was significantly higher than that of the CG in the second round (by $2.13 \%$ and $4.14 \%$ for the PTG) and the third round (by $4.75 \%$ and $7.71 \%$ for the PTG). In other words, plyometric training can delay the decrease in MDF, but it cannot alter the development of fatigue.

The ANCOVA results show that the BA of the PTG improved significantly after six weeks of training. Although not reflected as statistically significant, the BA increased as the number of rounds increased. These results show that the change occurred more gradually in the PTG. With respect to the average percentage of improvement, the PTG improved by $6.26 \%$ after six weeks of interventional training, whereas the CG improved by only $0.48 \%$. In volleyball, blocking is not only a defensive skill but also a skill essential for scoring. In team sports, the defensive ability is considered to be a display of individual agility $[28,29]$. Agility is an important index in team sports and therefore must be improved through functional training. Improving the ability of the neuromuscular system to adapt and control may be factors that improve BA. Plyometric training can stimulate the CNS signal transmission, which can improve the stretch-shortening cycle ability in the leg muscles. Through the training process, exercise patterns involving the ability to change directions in a short period of time can produce the effect of muscle stretching-contraction-circulation, yielding the benefits of reflexive muscle strength and stretch reflex characteristics and improving the instant reaction ability.

The ANCOVA results showed that the standing jump height in the PTG after six weeks of training was significantly higher than that of the CG, increasing by $2.23 \mathrm{~cm}(3.33 \%)$ in the PTG and slightly decreasing by $0.07 \mathrm{~cm}(-0.10 \%)$ in the CG. Although the improvement in the PTG was only approximately $2 \mathrm{~cm}$, a difference in height of $2 \mathrm{~cm}$ is a sign of approaching the ability to break through the maximum limits of the body in players performing at high levels. The results of the previous studies have shown that undergoing plyometric training two to three times a week can effectively improve the maximum output power and the rebounding ability of muscles [30], and the results of the present study are consistent with those of previous studies.

The results from this study show that repeated jumping causes fatigue-related declines in the RFM and TAM. In particular, blocking jumps require fast SSC muscle activity. Because of the lower leg muscle activity in the braking phase, the muscle stiffness decreases and simultaneously diminishes the efficacy of SSC actions [31,32]. To achieve the maximum rebound in muscle elastic strain energy during the push-off phase, the muscle must maintain a high stiffness during the braking phase [33]. Therefore, the EMG activities change during the jump, depending on the stiffness of the muscle. In this study, the power spectrum of MDFs (RFM and TAM) was biased towards low frequencies. The blocking agility test in this experiment comprised 14 blocking jumps, which required the athletes to perform repeated SSC movements, which in turn led to declines in muscle activities. In this research, a six-week plyometric training period was applied to provide additional loads on the athletes and thereby stimulate adaptation. At the same time, the training program included sufficient rest time, which is important for the recovery of muscle functions, thereby delaying the occurrence of muscle fatigue and improving the CNS function after the training period. In addition, enhancements in the acquired abilities through plyometric training primarily result from the stimulation of the muscular and nervous systems, and the process of adaptation.

The athletes recruited in this study were sufficiently proficient and physically fit to fulfill the requirements of volleyball matches. While we found effective improvements in these volleyball players after plyometric training, we produced no conclusive results regarding the settings that should be used for optimal effects. Further studies should help determine the appropriate intensity of plyometric 
training. The results of this study may not be generalizable to populations differing from the skill level, sex and age. In this study, a potential weakness of the data collection method was that the BA test program included a fixed exercise intensity. However, the exercise intensity in real games is variable; therefore, the time intervals could be varied in future studies.

\section{Conclusions}

Based on the results of this study, six weeks of appropriate plyometric training can delay the decrease in MDF in the RFM and TAM, improve vertical jump height, and significantly shorten BA in volleyball players when movement and jumping are combined. Increasing the muscle strength and coordination of movements in the legs facilitates rapid and complete movements during a moving block; that is, the total power output during the nerve conduction and muscle contraction processes is improved. For volleyball athletes, jumping high and fast and maintaining a high level of performance on the court is the goal of training. Based on fundamental training principles (e.g., variation, periodicity, individualization), recommendations to coaches include the use of different jumping loads to stimulate adaptation in the athletes. Neuromuscular fatigue during continuous jumping can be evaluated using the MDF after EMG spectrum analysis. Such markers can therefore be used to assess the fatigue resistance of athletes in competition-like situations.

Author Contributions: Conceptualization, M.-H.W. and K.-C.C.; methodology, M.-H.W.; software, C.-S.H.; validation, M.-H.H., C.-Y.C. and K.-C.L.; formal analysis, M.-H.H.; investigation, M.-H.W.; resources, K.-C.C.; data curation, C.-H.C.; writing-original draft preparation, C.-Y.C.; writing—review and editing, K.-C.L.; visualization, K.-C.L.; supervision, M.-H.W.; project administration, C.-S.H. All authors have read and agreed to the published version of the manuscript.

Funding: This research received no external funding.

Conflicts of Interest: The authors declare no conflict of interest.

\section{References}

1. Sheppard, J.M.; Gabbett, T.; Taylor, K.L.; Dorman, J.; Lebedew, A.J.; Borgeaud, R. Development of a repeated-effort test for elite men's volleyball. Int. J. Sport Physiol. 2007, 2, 292-304. [CrossRef]

2. Sheppard, J.; Borgeaud, R.; Strugnel, A. Influence of stature on movement speed and repeated efforts in elite volleyball players. J. Aust. Strength Cond. 2008, 16, 12-14.

3. Sheppard, J.M.; Cronin, J.B.; Gabbett, T.J.; McGuigan, M.R.; Etxebarria, N.; Newton, R.U. Relative importance of strength, power, and anthropometric measures to jump performance of elite volleyball players. J. Strength Cond. Res. 2008, 22, 758-765. [CrossRef]

4. Menzel, H.J.; Chagas, M.H.; Szmuchrowski, L.A.; Araujo, S.R.; Campos, C.E.; Giannetti, M.R. Usefulness of the jump-and-reach test in assessment of vertical jump performance. Percept. Mot. Skills. 2010, 110, 150-158. [CrossRef]

5. Merletti, R.; Parker, P. Electromyography—Physiology, Engineering and Noninvasive Applications, 1st ed.; John Wiley \& Sons Inc.: Hoboken, NJ, USA, 2004.

6. Bojsen-Møller, J.; Magnusson, S.P.; Rasmussen, L.R.; Kjaer, M.; Aagaard, P. Muscle performance during maximal isometric and dynamic contractions is influenced by the stiffness of the tendinous structures. J. Appl. Physiol. 2005, 99, 986-994. [CrossRef]

7. Ishikawa, M.; Komi, P.V.; Finni, T.; Kuitunen, S. Contribution of the tendinous tissue to force enhancement during stretch-shortening cycle exercise depends on the prestretch and concentric phase intensities. J. Electromyogr. Kines. 2006, 16, 423-431. [CrossRef]

8. Bobbert, M.F.; Van Der Krogt, M.M.; Van Doorn, W.H.; de Ruiter, C.J. Effects of Fatigue of Plantarflexors on Control and Performance in Vertical Jumping. Med. Sci. Sports Exerc. Off. J. Am. Coll. Sports Med. 2011, 43, 673-684. [CrossRef]

9. Horita, T.; Komi, P.V.; Nicol, C.; Kyrolainen, H. Effect of exhausting stretch-shortening cycle exerciseon the time course of mechanical behaviour in the drop jump: Possible role of muscle damage. Eur. J. Appl. Physiol. 1999, 79, 160-167. [CrossRef] 
10. Horita, T.; Komi, P.; Hämäläinen, I.; Avela, J. Exhausting stretch-shortening cycle (SSC) exercise causes greater impairment in SSC performance than in pure concentric performance. Eur. J. Appl. Physiol. 2003, 88, 527-534. [CrossRef]

11. Kuitunen, S.; Avela, J.; Kyrolainen, H.; Nicol, C.; Komi, P.V. Acute and prolonged reduction in joint stiffness in humans after exhausting stretch-shortening cycle exercise. Eur. J. Appl. Physiol. 2002, 88, 107-116.

12. Neyroud, D.; Samararatne, J.; Kayser, B.; Place, N. Neuromuscular Fatigue After Repeated Jumping With Concomitant Electrical Stimulation. Int. J. Sport Physiol. 2017, 12, 1335-1340. [CrossRef] [PubMed]

13. ve Dikey, A.; Şimşek, D.; Kırkaya, İ.; Güngör, E.O.; Soylu, A.R. Relationships among Vertical Jumping Performance, EMG Activation, and Knee Extensor and Flexor Muscle Strength in Turkish Elite Male Volleyball Players. Turk. Klin. J. Sports Sci. 2016, 8, 46-56.

14. Pereira, G.; de Freitas, P.B.; Rodacki, A.; Ugrinowitsch, C.; Fowler, N.; Kokubun, E. Evaluation of an innovative critical power model in intermittent vertical jump. Int. J. Sports Med. 2009, 30, 802-807. [CrossRef] [PubMed]

15. Pereira, G.; Correia, R.; Ugrinowitsch, C.; Nakamura, F.; Rodacki, A.; Fowler, N.; Kokubun, E. The rating of perceived exertion predicts intermittent vertical jump demand and performance. J. Sports Sci. 2011, 29, 927-932. [CrossRef]

16. Skurvydas, A.; Brazaitis, M.; Streckis, V.; Rudas, E. The effect of plyometric training on central and peripheral fatigue in boys. Int. J. Sports Med. 2010, 31, 451-457. [CrossRef]

17. Nordlund, M.M.; Thorstensson, A.; Cresswell, A.G. Central and peripheral contributions to fatigue in relation to level of activation during repeated maximal voluntary isometric plantar flexions. J. Appl. Physiol. 2004, 96, 218-225. [CrossRef]

18. Makaruk, H.; Sacewicz, T. Effects of plyometric training on maximal power output and jumping ability. Hum. Mov. 2010, 11, 17-22. [CrossRef]

19. Markovic, G.; Mikulic, P. Neuro-musculoskeletal and performance adaptations to lower-extremity plyometric training. Sports Med. 2010, 40, 859-895. [CrossRef]

20. Kim, Y.Y.; Park, S.E. Comparison of whole-body vibration exercise and plyometric exercise to improve isokinetic muscular strength, jumping performance and balance of female volleyball players. J. Phys. Ther. Sci. 2016, 28, 3140-3144. [CrossRef]

21. Pereira, A.M.; Costa, A.; Santos, P.; Figueiredo, T.; Vicente João, P. Training strategy of explosive strength in young female volleyball players. Medicina 2015, 51, 126-131. [CrossRef]

22. Wulf, G.; Dufek, J.S.; Lozano, L.; Pettigrew, C. Increased jump height and reduced EMG activity with an external focus. Hum. Mov. Sci. 2010, 29, 440-448. [CrossRef] [PubMed]

23. Ho, C.S.; Lin, K.C.; Chen, K.C.; Chiu, P.K.; Chen, H.J. System design and application for evaluation of blocking agility in volleyball. Proc. Inst. Mech. Eng. Part P J. Sports Eng. Technol. 2016, 230, 195-202. [CrossRef]

24. Piper, T.J.; Erdmann, L.D. A 4 step plyometric program. Strength Cond. J. 1998, 20, 72-73. [CrossRef]

25. Hayashibe, M.; Zhang, Q.; Guiraud, D.; Fattal, C. Evoked EMG-based torque prediction under muscle fatigue in implanted neural stimulation. J. Neural Eng. 2011, 8, 064001. [CrossRef] [PubMed]

26. Stulen, F.B.; De Luca, C.J. Frequency parameters of the myoelectric signal as a measure of muscle conduction velocity. IEEE Trans. Biomed. Eng. 1981, 7, 515-523. [CrossRef]

27. Merletti, R.; Conte, L.R.L. Surface EMG signal processing during isometric contractions. J. Electromyogr. Kines. 1997, 7, 241-250. [CrossRef]

28. Sheppard, J.M.; Young, W.B.; Doyle, T.L.; Sheppard, T.A.; Newton, R.U. An evaluation of a new test of reactive agility and its relationship to sprint speed and change of direction speed. J. Sci. Med. Sport. 2006, 9, 342-349. [CrossRef]

29. Farrow, D.; Young, W.; Bruce, L. The development of a test of reactive agility for netball: A new methodology. J. Sci. Med. Sport. 2005, 8, 52-60. [CrossRef]

30. Potteiger, J.A.; Lockwood, R.H.; Haub, M.D.; Dolezal, B.A.; Alumzaini, K.S.; Schroeder, J.M.; Zebas, C.J. Muscle power and fiber characteristic following 8 weeks of plyometric training. J. Strength Cond. Res. 1999, 13, 275-279. 
31. Arampatzis, A.; Stafilidis, S.; Morey-Klapsing, G.; Brüggemann, G.P. Interaction of the human body and surfaces of different stiffness during drop jumps. Med. Sci. Sports Exerc. 2004, 36, 451-459. [CrossRef]

32. Lesinski, M.; Prieske, O.; Demps, M.; Granacher, U. Effects of fatigue and surface instability on neuromuscular performance during jumping. Scand J. Med Sci Spor. 2016, 26, 1140-1150. [CrossRef] [PubMed]

33. Avela, J.; Komi, P.V.; Santos, P.M. Effects of differently induced stretch loads on neuromuscular control in drop jump exercise. Eur. J. Appl. Physiol. Occup. Physiol. 1996, 72, 553-562. [CrossRef] [PubMed]

(C) 2020 by the authors. Licensee MDPI, Basel, Switzerland. This article is an open access article distributed under the terms and conditions of the Creative Commons Attribution (CC BY) license (http://creativecommons.org/licenses/by/4.0/). 